\title{
Editorial: Modern Tools for Time-Resolved Luminescence Biosensing and Imaging
}

\author{
Yiqing $\mathrm{Lu}^{1 *}$, Gerard Marriott ${ }^{2 *}$ and Klaus Suhling ${ }^{3 *}$ \\ ${ }^{1}$ School of Engineering, Macquarie University, Sydney, NSW, Australia, ${ }^{2}$ Department of Bioengineering, University of California \\ Berkeley, Berkeley, CA, United States, ${ }^{3}$ Department of Physics, King's College London, London, United Kingdom
}

Keywords: time-resolved luminescence, luminescence lifetime, exponential decay, fluorescence lifetime imaging (FLIM), Förster resonance energy transfer (FRET), time-correlated single photon counting (TCSPC)

\section{Editorial on the Research Topic}

\section{Modern Tools for Time-Resolved Luminescence Biosensing and Imaging}

The time evolution of luminescence decay, often described by the parameter of luminescence lifetime assuming first-order kinetics, is among the key characteristics of a luminescence substance. This makes time-resolved luminescence measurement a powerful tool for identifying substances of interest, especially in biological samples where differentiation based on luminescence colors may be inadequate or ineffective [1-3]. Moreover, for quantitative luminescence intensity measurements, the concentration in biological samples is hard to control, and typically needs a calibration, whereas

OPEN ACCESS

Edited by:

Lorenzo Pavesi,

University of Trento, Italy

*Correspondence:

Yiqing Lu

yiqing.lu@mq.edu.au

Gerard Marriott

gmlab@berkeley.edu

Klaus Suhling

klaus.suhling@kcl.ac.uk

Specialty section:

This article was submitted to

Optics and Photonics,

a section of the journal

Frontiers in Physics

Received: 15 June 2021

Accepted: 15 June 2021

Published: 29 June 2021

Citation:

Lu Y, Marriott G and Suhling K (2021) Editorial: Modern Tools for Time-

Resolved Luminescence Biosensing and Imaging.

Front. Phys. 9:725234.

doi: 10.3389/fphy.2021.725234 the luminescence decay is largely independent of the luminophore concentration. The kinetics of luminescence decay is also influenced by the environment, enabling various sensing applications of time-resolved luminescence in biology and medicine $[4,5]$.

In this multidisciplinary Research Topic, a collection of six Original Research, three Brief Research Report, one Technology and Code, and three Review articles are presented, featuring some of the latest advances in time-resolved luminescence instruments, probes, data processing, interpretation, and applications for biosensing and imaging.

Depending on the timescale of the luminescence decay, which varies from nanoseconds for typical fluorophores to seconds and longer for some phosphors, time-resolved luminescence measurement encompasses a diverse range of techniques, probes and devices suited for different temporal resolution. Hirvonen and Suhling provide a detailed overview of the fast timing underpinning fluorescence lifetime imaging (FLIM) - a technique which celebrated its 30th anniversary recently [6], and highlight a few recent examples showcasing applications in life sciences. Meanwhile, Deng et al. survey techniques for measuring long-lived emission, with particular focus on methods using mechanical choppers. Special attention is paid to automatic synchronization based on the same chopper acting as both the pulse generator and the detection shutter, with detailed discussion on their advantages, challenges, and future development prospects. Generally speaking, time-resolved measurement takes longer than steady-state measurement to perform in order to capture the time evolution at the cost of analytical throughput. Bitton et al. review the recent advancements in microscopy and flow cytometry for high-throughput lifetime measurements, and provide their outlook on future developments integrating machine learning.

On the other hand, regardless of the temporal resolution, common principles and methods are applicable to time-resolved luminescence measurement, which can be performed either in the time domain or in the frequency domain [7]. On the former, Ziniuk et al. implement a rapid lifetime determination technique for lifetime imaging of Er-doped nanoparticles emitting shortwave infrared luminescence, which has the potential for in vivo bioimaging. Li et al. compare the outputs of several 
lifetime determination algorithms when the fluorescence follows a multi-exponential decay. They further propose using the ratio of intensity and amplitude-weighted average lifetimes as a new indicator of energy transfer efficiency. On the latter, Sambrano Jr et al. report their progress on developing a flow cytometry method capable of frequency-domain lifetime measurement, based on an acoustic-focusing microfluidic chip to control the flow speed. Sumetsky et al. examine the lifetimes calculated from frequency-domain FLIM, showing a simple linear relationship to the amplitude-weighted lifetime associated with energy transfer efficiency. For calibration purposes, Xiao et al. propose a modified (mirror-symmetric) method to obtain a synthetic instrument response function (IRF) from time-correlated single photon counting data, which offers better performance than the traditional (differential) synthetic IRF for lifetime analysis. In terms of data processing and interpretation, Haas et al. present a multidimensional phasor demixing method for analyzing data from spectral-resolved FLIM microscopy, which is applied to multiplexed biosensing of the cell-death proteases Caspase-2, -3 and -9 on the single cell level.

The versatility of time-resolved luminescence methods keeps inspiring new applications. Mould et al. apply two-photon FLIM microscopy to investigate the effect of cannabidiol on breast cancer cells. They show that at increased concentration of cannabidiol, mitochondrial bound $\mathrm{NAD}(\mathrm{P}) \mathrm{H}$ decreases significantly and correlates to increased oxidative stress. Chorvatova et al. employ endogenous fluorescence of water moss in response to several heavy

\section{REFERENCES}

1. Soini E, Lövgren T, and Reimer CB. Time-Resolved Fluorescence of Lanthanide Probes and Applications in Biotechnology. Crit Revs. Anal Chem (1987) 18: 105-54. doi:10.1080/10408348708085565

2. Marriott G, Clegg RM, Arndt-Jovin DJ, and Jovin TM. Time Resolved Imaging Microscopy. Phosphorescence and Delayed Fluorescence Imaging. Biophysical J (1991) 60:1374-87. doi:10.1016/s0006-3495(91)82175-0

3. Fan Y, Wang P, Lu Y, Wang R, Zhou L, Zheng X, et al. Lifetime-engineered NIRII Nanoparticles Unlock Multiplexed In Vivo Imaging. Nat Nanotech (2018) 13: 941-6. doi:10.1038/s41565-018-0221-0

4. Berezin MY, and Achilefu S. Fluorescence Lifetime Measurements and Biological Imaging. Chem Rev (2010) 110:2641-84. doi:10.1021/cr900343z

5. Suhling K, Hirvonen LM, Levitt JA, Chung P-H, Tregidgo C, Le Marois A, et al. Fluorescence Lifetime Imaging (FLIM): Basic Concepts and Some Recent Developments. Med Photon (2015) 27:3-40. doi:10.1016/j.medpho.2014.12.001 metal nanoparticles to demonstrate that the fluorescence lifetime of the endogenous fluorescence is a sensitive parameter for detecting environmental pollution. Gallian et al. present an immunoassay platform based on Mn-doped quantum dots and a compact plate reader equipped with a pulsed laser diode and a time-gated detector. They achieve sensitive detection of the capsular polysaccharide from Burkholderia pseudomallei (a Tier 1 selected agent) with a detection limit two orders of magnitude lower than steady-state measurements, which can be advantageous for in-field diagnosis. Bhartiya et al. summarize a detailed protocol for multicolour fluorescence in situ hybridization combined with DAPI FLIM, allowing study of the structural differences in the 46 chromosomes of the human genome.

We thank all of our contributing authors for their dedication to the Research Topic, and the reviewers for their constructive comments and suggestions. It is hoped that the Research Topic will serve as a useful reference work for a broad audience, encouraging cross-disciplinary collaborations, and accelerating future advances and innovation in Time-Resolved Luminescence Biosensing and Imaging technologies and their applications in the biological, environmental, and health sciences.

\section{AUTHOR CONTRIBUTIONS}

All authors listed have made a substantial, direct, and intellectual contribution to the work and approved it for publication.

6. Ameer-Beg S, Suhling K, and Kuimova M. Special Issue on Fluorescence Lifetime Imaging (FLIM): from Fundamentals to Applications. Methods Appl Fluoresc (2020) 8:040401. doi:10.1088/2050-6120/abad19

7. Lakowicz JR. Principles of Fluorescence Spectroscopy. 3rd ed. New York: Springer US (2007).

Conflict of Interest: The authors declare that the research was conducted in the absence of any commercial or financial relationships that could be construed as a potential conflict of interest.

Copyright (c) $2021 \mathrm{Lu}$, Marriott and Suhling. This is an open-access article distributed under the terms of the Creative Commons Attribution License (CC $B Y)$. The use, distribution or reproduction in other forums is permitted, provided the original author(s) and the copyright owner(s) are credited and that the original publication in this journal is cited, in accordance with accepted academic practice. No use, distribution or reproduction is permitted which does not comply with these terms. 